\title{
Low-Resistance Molecular Wires Propagate Spin-Polarized Currents
}

\author{
George Bullard, ${ }^{\dagger}$ Francesco Tassinari, ${ }^{\ddagger}$ Chih-Hung Ko, ${ }^{\dagger}$ Amit Kumar Mondal, ${ }^{\ddagger}$ Ruobing Wang, ${ }^{\dagger}$

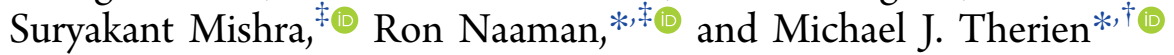

${ }^{\dagger}$ Department of Chemistry, Duke University, Durham, North Carolina 27708, United States

${ }^{\ddagger}$ Department of Chemical and Biological Physics, Weizmann Institute of Science, Rehovot 76100, Israel

\section{Supporting Information}

\begin{abstract}
Spin based properties, applications, and devices are typically related to inorganic ferromagnetic materials. The development of organic materials for spintronic applications has long been encumbered by its reliance on ferromagnetic electrodes for polarized spin injection. The discovery of the chirality-induced spin selectivity (CISS) effect, in which chiral organic molecules serve as spin filters, defines a marked departure from this paradigm because it exploits soft materials, operates at ambient temperature, and eliminates the need for a magnetic electrode. To date, the CISS effect has been explored exclusively in molecular insulators. Here we combine chiral molecules, which serve as spin filters, with molecular wires that despite not being chiral, function to preserve spin polarization. Self-assembled monolayers (SAMs) of right-handed helical (L-proline $)_{8}\left(\mathbf{P r o}_{8}\right)$ and corresponding peptides, N-terminal conjugated to (porphinato) zinc or meso-to-meso ethyne-bridged (porphinato)zinc structures ( $\operatorname{Pro}_{8} \mathbf{P Z} \mathbf{n}_{n}$ ), were interrogated via magnetic conducting atomic force microscopy (mC-AFM), spin-dependent electrochemistry, and spin Hall devices that measure the spin polarizability that accompanies the charge polarization. These data show that chiral molecules are not required to transmit spin-polarized currents made possible by the CISS mechanism. Measured Hall voltages for $\mathbf{P r o}_{8} \mathbf{P Z n}_{1-3}$ substantially exceed that determined for the Pro control and $_{8}$ increase dramatically as the conjugation length of the achiral $\mathbf{P Z \mathbf { n } _ { n }}$ component increases; $\mathrm{mC}$-AFM data underscore that measured spin selectivities increase with an increasing Pro $\mathbf{P Z n}_{1-3}$ N-terminal conjugation. Because of these effects, spindependent electrochemical data demonstrate that spin-polarized currents, which trace their genesis to the chiral Pro $\mathbf{s}_{8}$ moiety, propagate with no apparent dephasing over the augmented $\mathbf{P r o}_{8} \mathbf{P Z n _ { n }}$ length scales, showing that spin currents may be transmitted over molecular distances that greatly exceed the length of the chiral moiety that makes possible the CISS effect.
\end{abstract}

\section{INTRODUCTION}

Molecular electronics promises both new opportunities for device miniaturization and possibilities for revolutionary device concepts. For example, organic spintronics has garnered significant attention since the discovery of the giant magnetoresistance effect in $\mathrm{Alq}_{3}$ films sandwiched between ferromagnetic (FM) electrodes. Leveraging charge carrier spins in organic material-based devices defines an appealing approach to processing and carrying information at high frequencies and low voltages. ${ }^{2}$ However, realizing organic spintronic devices is hindered by the complexity of making organic-inorganic contacts, underscored by the need to inject spin-polarized electrons from inorganic FMs into organic molecules. Although organic semiconductors (OSCs) can provide intrinsically low spin-orbit coupling, conventional OSCs provide only modest spin free mean path lengths. ${ }^{3-10}$ Furthermore, the Schottky barrier between the FM and the OSC can cause spin depolarization, which limits the spin injection efficiency. ${ }^{11}$

The chirality-induced spin selectivity (CISS) effect, ${ }^{12-15}$ in which chiral organic molecules serve as spin filters, opens new possibilities in organic spintronics because it operates at ambient temperature and paves the way to generating spin currents in the absence of an FM. The CISS effect arises from a coupling between the spin and the linear momentum of an electron via the chiral electrostatic potential of the molecule. In the electron's rest frame, the current transmitted through the chiral molecule generates a magnetic field proportional to the velocity of the moving electron and the electric field operating on the electron. This effective magnetic field acts on the electron's magnetic moment, stabilizing one spin state and destabilizing the other. For this reason, the tunneling probability of an electron through a chiral system depends on its spin. ${ }^{13}$

Although the CISS effect has been demonstrated in selfassembled monolayers (SAMs) composed of a wide range of chiral structures that include low-molecular-weight molecules, ${ }^{16}$ DNA, ${ }^{13,14,17,18}$ oligopeptides, ${ }^{19}$ and proteins, ${ }^{20}$ spinpolarized currents realized by this mechanism have thus far been transmitted exclusively through chiral molecular insulators. Theoretical treatments, however, suggest the central importance of coherent charge transmission and resonant tunneling in determining CISS effect magnitudes. ${ }^{21}$ A related and equally important issue for the development of CISS-based spintronics concerns factors that drive spin current dephasing.

Received: June 13, 2019

Published: August 14, 2019 
Namely, once a spin current has been generated via tunneling through a chiral molecule, to what extent may it persist through an appended achiral structure?

Figure 1a depicts a family of compositions fabricated to probe these issues. These structures all feature a poly $(\mathrm{L}-$

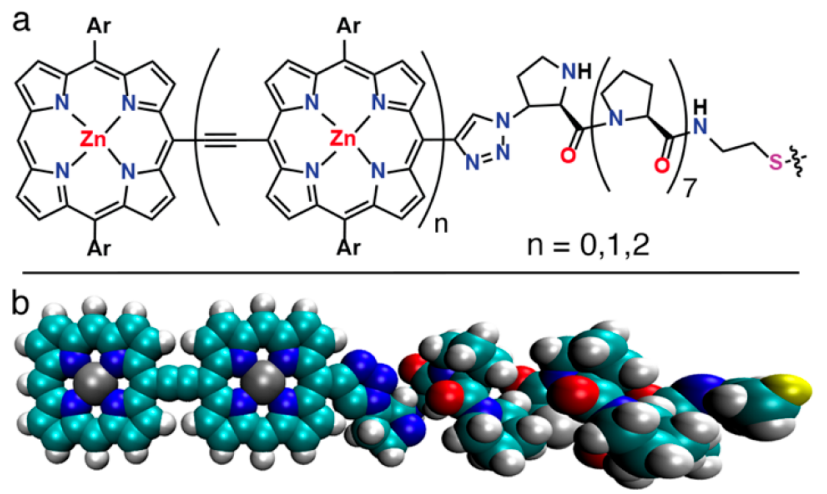

Figure 1. (a) Structures of $\mathbf{P r o}_{8} \mathbf{P Z n}_{n}$ molecules in which the thiolterminated $\mathrm{Pro}_{8}$ unit adopts the right-handed PPI helical structure. Ar $=2^{\prime}, 6^{\prime}$-bis(3,3-dimethyl-1-butyloxy)phenyl. (b) Structure of Pro $_{8} \mathbf{P Z n}_{2}$ with van der Waals spheres at $90 \%$.

proline) unit ( Pro $\left._{7}\right)$ that adopts a polyproline I, right-handed helical twist (Figure 1b) in which the C-terminus has been conjugated to 2-amino-ethanethiol. $\mathrm{N}$-terminal coupling of (4R)-azido-L-proline to $\mathrm{Pro}_{7}$ provides the Pro $_{8}$ peptide with functionality for click-mediated conjugation to meso-ethynylterminated (porphinato)zinc and multi-[(porphinato)zinc] structures $\left(\mathbf{P Z n} \mathbf{n}_{1-3}\right)$ to give $\mathbf{P r o}_{\mathbf{8}} \mathbf{P Z n} \mathbf{n}_{\mathbf{1 - 3}}$ (Figure 1; Supporting Information).

\section{EXPERIMENTAL SECTION}

A description of the synthesis and characterization of all new compounds, detailed reaction schemes, device design, fabrication, and characterization, methods used to prepare and characterize selfassembled monolayers, and instrumentation utilized in this study may be found in the Supporting Information.

\section{RESULTS AND DISCUSSION}

$\mathbf{P Z n}_{2}$ and $\mathbf{P Z n _ { 3 }}$ belong to a larger class of meso-to-meso ethyne-bridged (porphinato)zinc(II) structures ( $\mathbf{P Z \mathbf { n } _ { n }}$ oligomers) that manifest exceptional electro-optical properties that include substantial polarizabilities and low-lying singlet states that are aligned exclusively along the long molecular axis. $^{6-10}$ ESR data acquired for charge-doped $\mathbf{P Z n _ { n }}$ demonstrate globally delocalized hole and electron polaron states through substantial oligomer lengths $s^{4,5}$ and corresponding spin relaxation times 3 orders of magnitude greater than that established for classic organic semiconductor $\mathrm{Alq}_{3}{ }^{22}$ Scanning tunneling microscopy break-junction measurements show that metal-(thiol-PZn ${ }_{n}$-thiol)-metal junctions express quasi-ohmic resistances and afford one of the lowest $\beta$ values $(\beta=0.034$ $\AA^{-1}$; i.e., the phenomenological resistance decay parameter across the barrier) yet determined for thiol-terminated single molecules. ${ }^{23}$ Corresponding measurements, made for bulk molecular electronic devices fabricated from monolayers of these molecules, highlight identical distance-dependent resistances. $^{24}$ Collectively, these properties suggest the potential utilty of $\mathbf{P Z \mathbf { n } _ { n }}$ to suppress spin randomization following the production of a polarized spin current via the CISS mechanism in a device configuration.
Self-assembled monolayers (SAMs) of $\mathbf{P r o}_{8}$ and $\mathbf{P r o}_{\mathbf{8}} \mathbf{P Z n}_{1-3}$ were adsorbed on a Au-coated GaN/AlGaN two-dimensional electron gas (2DEG) structure that is integrated into a spin polarization Hall device configuration (Figure 2a). ${ }^{25,26}$ The

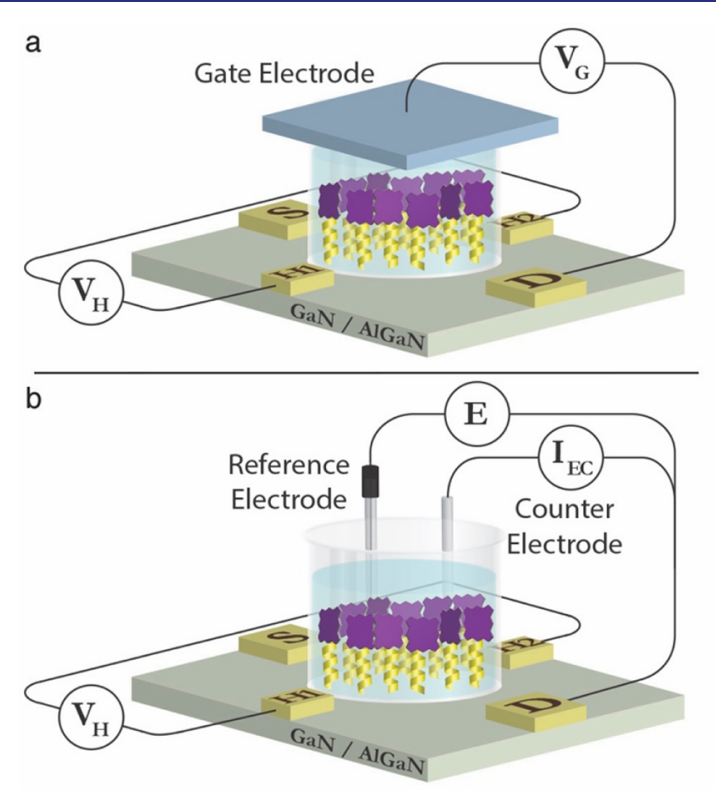

Figure 2. (a) Schematic of the spin polarization Hall device ${ }^{24,25}$ used to measure the charge polarization-induced spin polarization driven by the chiral $\mathrm{Pro}_{8}$ and $\mathbf{P r o}_{8} \mathbf{P Z n}_{1-3}$ SAMs. The top gate electrode (blue) is used to apply an electric field. The electrode is insulated from the electrolyte solution; therefore, no faradaic current flows between the gate and the Hall device. (b) Corresponding spindependent electrochemical (SDE) device configuration where the monolayer-covered Hall bar functions as the working electrode.

SAM preparation was performed under conditions in which the Pro $_{8}$ unit adopts a right-handed polyproline I helix (Figure 1b) ${ }^{27} \mathrm{~A}$ constant current/voltage is driven between the source (S) and drain (D) electrodes through the device, and the Hall voltage is measured between the $\mathrm{H} 1$ and $\mathrm{H} 2$ electrodes along the direction perpendicular to the current flow. ${ }^{25,26}$ By applying an electric field between the gate electrode and the device, the SAM is charge-polarized. If this charge polarization is accompanied by spin polarization, then a Hall voltage is measured. ${ }^{26}$ Spin-dependent electrochemical (SDE) measurements may also be carried out using the same device when the 2DEG structure is configured as the working electrode in a standard three-electrode electrochemical cell. ${ }^{25}$ While the cyclic voltammetric $(\mathrm{CV})$ response is measured, the Hall potential is monitored simultaneously, providing information regarding the spin selectivity of the redox reaction. The Hall voltages result from the continuous nonequilibrium spinpolarized current that flows from the Au-coated GaN/AlGaN layer through the SAMs. The accumulated spin polarization at the $\mathrm{GaN} / \mathrm{AlGaN} / \mathrm{SAM}$ interface generates a magnetic field capable of being detected by the Hall devices. The measured layer heights of the SAMs in these devices range between 11 (Pro ${ }_{8}$ ) and $27 \AA$ (Pro $\mathbf{P Z n}_{3}$ ) (Supporting Information).

The spin polarization that accompanies charge polarization in $\mathbf{P r o}_{8}$ and Pro $_{8} \mathbf{P Z n}_{1-3}$ SAMs was measured using sequential gate pulses from 10 to $10 \mathrm{~V}$ at $2 \mathrm{~V}$ intervals (Figure $3 \mathrm{a}$ ). Note that relative to $\mathbf{P r o}_{8}$ monolayers, $\mathbf{P r o}_{8} \mathbf{P Z n}_{1}$ SAMs, in which an achiral PZn unit is covalently attached to the $\mathrm{N}$-terminal 

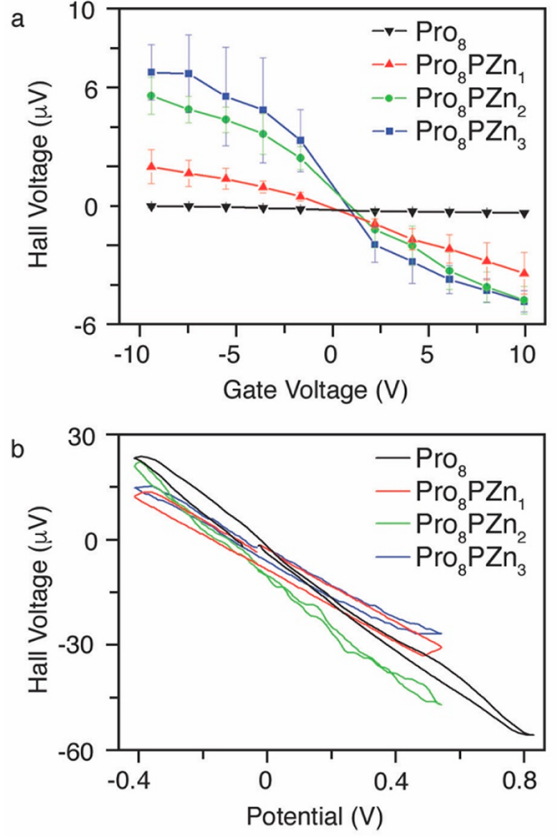

Figure 3. Polarization experiments performed on spin-Hall devices (Figure 2) as a function of monolayer composition. (a) Hall potential measured as a function of gate voltage for $\mathbf{P r o}_{8^{-}}$and $\mathbf{P r o}_{8} \mathbf{P Z n}_{1-3^{-}}$ based devices. (b) Hall potential measured concurrently with CV responses recorded during the SDE experiments. The slopes of the $\mathrm{CV}$ responses are consistent across these $\mathbf{P r o}_{8^{-}}$and $\mathbf{P r o}_{8} \mathbf{P Z n}_{1-3^{-}}$ SAM-based devices, indicating that spin polarization has been unaffected by $\mathbf{P Z n} \mathbf{n}_{n}$ conjugation to the $\mathbf{P r o}_{8}$ oligomer. All measurements were carried out in $0.1 \mathrm{M}$ PBS buffer. SDE experimental conditions: potentiometric window $=-0.4$ to $0.8 \mathrm{~V}$; scan rate $=50$ $\mathrm{mV} / \mathrm{s}$; working electrode $=$ Hall bar; counter electrode $=$ Pt wire; reference electrode $=\mathrm{Ag}$ wire.

peptide of the $\mathrm{Pro}_{8}$ unit, have no influence on the nature of the observed spin-selective polarization. Instead, $\mathbf{P r o}_{8} \mathbf{P Z n _ { 1 }}$ manifests more than an order of magnitude amplification of the observed spin polarization relative to the corresponding $\mathbf{P r o}_{8}$ monolayer. Furthermore, as the $\mathbf{P r o}_{8}$ terminal conjugation increases, the spin polarization magnitude is progressively amplified (Figure 3a). These Figure 3a data are compelling in multiple respects. (i) As polarization modulation-infrared reflection-adsorption spectroscopy (PM-IRRAS) and ellipsometry data demonstrate, Hall bar loading densities for these Pro $_{8} \mathbf{P Z n}_{n}$ SAMs are essentially identical to those determined for benchmark CISS studies of 30 base pair double-stranded DNA (30bp-dsDNA; Supporting Information). ${ }^{28}$ It is noteworthy that the Hall voltage response measured for $\mathbf{P r o}_{\mathbf{8}} \mathbf{P Z n _ { 2 }}$ exceeds that for $30 \mathrm{bp}$-dsDNA in identical devices. Because 30bp-dsDNA spans a molecular length scale of $>100 \AA$, these data show that Hall potentials of this magnitude may be realized over modest molecular distances $\left(\mathbf{P r o}_{8} \mathbf{P Z n}_{2} \approx 35 \AA\right.$ ) while exploiting a chiral molecule $1 / 10$ as long as this ds-DNA benchmark. This effect likely originates in part from the fact that the phenomenological resistance decay parameter for charge tunneling through B-DNA is $\sim 0.64 \AA^{-1}, 29$ whereas the corresponding Pro $_{n} \beta$ value has been determined to be 0.15 $\AA^{-1} \cdot{ }^{30}$ (ii) The magnitudes of the Hall voltage responses measured as a function of gate voltage show an unprecedented dependence upon molecular length: Hall voltages determined for Pro $_{8} \mathbf{P Z n}_{1-3}$ substantially exceed that determined for the Pro $_{8}$ control and increase dramatically relative to the Pro benchmark as the conjugation length of the achiral $\mathbf{P Z} \mathbf{n}_{n}$ component increases. Furthermore, the magnitude of the observed Figure 3a sigmoidal response parallels the extent of conjugation. These data indicate that highly polarizable $\mathbf{P Z} \mathbf{n}_{n}$ structures, $^{4-6}$ despite lacking chirality, can dramatically augment the charge-polarization-induced spin polarization of chiral $\mathrm{Pro}_{8}$ molecules. These Figure $3 \mathrm{a}$ dependences are congruent with the established conjugation-length-dependent polarizabilities of $\mathbf{P Z n _ { n }}$ structures and the fact that the measured molecular resistances of these species, ordered at $\mathrm{Au}$ interfaces, track with valence band energies, which diminish with increasing molecular length. ${ }^{24}$
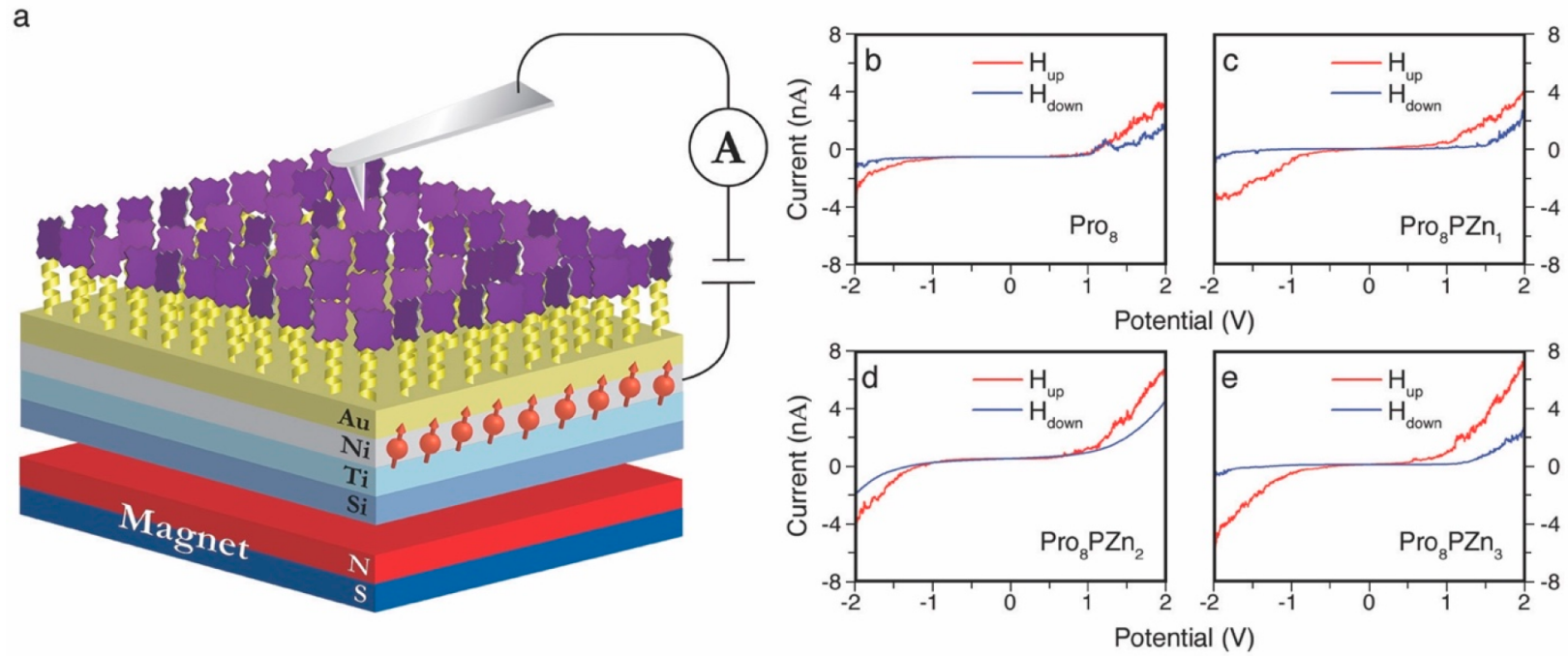

Figure 4. Schematic of the magnetic conducting atomic force microscopy (mC-AFM) experimental setup (a). A permanent magnet (0.3 T) was aligned with the substrate to polarize electron spins within the substrate Ni layer. Details regarding this experiment have been described previously (Supporting Information). ${ }^{14}$ Measurements were carried out on $\mathbf{P r o}_{8}$ and $\mathbf{P r o}_{8} \mathbf{P Z n}_{1-3}$ SAMs formed on Si wafers coated with successively thin layers of Ti/Ni/Au (10:120:10 nm). The four panels highlight the I-V responses acquired for (b) $\mathbf{P r o}_{8}$, (c) Pro $_{8} \mathbf{P Z n}_{1}$, (d) $\mathbf{P r o}_{8} \mathbf{P Z n}_{2}$, and (e) Pro $_{8} \mathbf{P Z n}_{3}$ over a -2 to $2 \mathrm{~V}$ potentiometric window; blue and red traces reflect the magnetic field orientation. 
SDE measurements, determined concurrently with these charge-polarization-induced spin polarization experiments with these Pro $_{8}$ and $\mathbf{P r o}_{8} \mathbf{P Z n}_{1-3}$ spin Hall devices (Figure $3 \mathrm{~b}$ ), further highlight the spin-polarizing utility of $\mathbf{P r o}_{8} \mathbf{P Z} \mathbf{n}_{n}$ compositions. Note the remarkable dependence of the Hall voltage on the Hall bar working electrode potential in which virtually superimposable responses are determined for Pro $_{8}$, $\mathbf{P r o}_{8} \mathbf{P Z n}_{1}, \mathbf{P r o}_{8} \mathbf{P Z n}_{2}$, and $\mathbf{P r o}_{8} \mathbf{P Z n}_{3}$. These data demonstrate that spin-polarized currents, which trace their genesis to the chiral Pro $\mathrm{Po}_{8}$ moiety, propagate with no apparent dephasing over augmented distances because of the agency of the $\mathbf{P Z n _ { n }}$ unit. Note in this regard that the $\mathbf{P Z n}_{3}$ length scale $(\sim 35 \AA)$

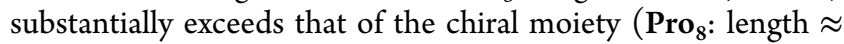
$15 \AA$ ), responsible for inducing the CISS effect. These results underscore the utility of meso-to-meso ethyne-bridged (porphinato)metal oligomers that possess long-spin relaxation times $^{22}$ and support global charge delocalization in spintronics applications. $^{5,22}$

Spin-polarized currents, generated via these $\mathbf{P r o}_{8} \mathbf{P Z n}_{n}$ SAMs, were also characterized using magnetic conducting atomic force microscopy (mC-AFM), a well-established method for measuring spin-selective conduction through molecular SAMs. ${ }^{14}$ In these experiments, the substrate is biased relative to the tip, which is grounded. Note that when the substrate is negatively biased, electrons are injected from the majority spin density of states, just below the Fermi level, and when the substrate is positively biased, spins are injected into the minority density of states just above the Fermi level. Hence, for the same substrate magnetization the electrons injected out have one spin whereas those that are injected in, from the monolayer, have the opposite spin. Pro $_{8}$ and $\mathrm{Pro}_{8} \mathrm{PZn}_{n}$ SAMs were formed on $\mathrm{Si}$ wafers coated with successively thin layers of $\mathrm{Ti} / \mathrm{Ni} / \mathrm{Au}(10: 120: 10 \mathrm{~nm})$. A permanent magnet $(\sim 0.3 \mathrm{~T})$, placed underneath the substrate, controls the spin alignment in the $\mathrm{Ni}$ substrate layer either parallel or antiparallel to the current direction. A Pt-coated AFM tip was used to measure the spin current passing through the SAMs as the substrate was biased potentiometrically between -2 and $2 \mathrm{~V}$ (Figure 4). Figure $4 \mathrm{~b}-\mathrm{e}$ displays the average $\mathrm{I}-\mathrm{V}$ responses in these $\mathrm{mC}$-AFM experiments obtained when the $\mathrm{Ni}$ spins are either pointing up or down; the Supporting Information provides the corresponding statistical data. Note that when these measurements are conducted in the absence of a magnetic field, the IV responses reflect a simple average of those obtained with the magnet pointing up and down. The nearly antisymmetric nature of the $\mathrm{I}-\mathrm{V}$ responses obtained for all of the $\mathbf{P r o}_{8}$ and $\mathbf{P r o}_{8} \mathbf{P Z n}_{n}$ SAMs in these mC-AFM experiments indicates that the conduction of one spin is dominant as current flows from the substrate to the AFM tip whereas the opposite spin is dominant as current flows in the other direction. These Figure 4 mC-AFM data corroborate (i) that the Pro 8 SAM spinpolarized current is maintained in corresponding $\mathbf{P r o}_{8} \mathbf{P Z n}_{n}$ SAMs and (ii) that these spin current conductance probabilities are modulated by the $\mathbf{P Z} \mathbf{n}_{n}$ conjugation length. The fact that spin selectivity increases with increasing conjugation length indicates that the difference between the effective barriers for the conduction of spin parallel or antiparallel to the electrons' velocity is amplified with augmented $\mathbf{P Z \mathbf { n } _ { n }}$ polarizability, consistent with the conjugation length- and valence band energy-dependent molecular resistances evaluated for these systems, ${ }^{23,24}$ and the extent of charge-polarization-induced spin polarization determined in the spin Hall device experiments (Figure 3a).

\section{CONCLUSIONS}

This work shows that once a spin-polarized current is generated by the CISS mechanism via charge tunneling though a chiral molecule, chiral structures are not required to further propagate this spin-polarized current. The combination of spin Hall device, spin-dependent electrochemical, and magnetic conducting atomic force microscopy data, acquired for these $\mathbf{P r o}_{\mathbf{8}}$ and $\mathbf{P r o}_{8} \mathbf{P Z n}_{n}$ systems, demonstrates that once a spin current has been generated via tunneling through a chiral oligoproline unit, it persists through the appended achiral $\mathbf{P Z n}_{n}$ structure, which, in contrast to Pro $_{8},{ }^{30}$ supports extensive charge delocalization ${ }^{4,5}$ and a low $\beta$ value $\left(\beta=0.034 \AA^{-1}\right.$; i.e., the phenomenological resistance decay parameter across the barrier). ${ }^{23}$ These Pro Prappended $^{- \text {ap }}$ $\mathbf{P Z} \mathbf{n}_{n}$ species suppress the spin randomization of the polarized spin current generated via the CISS mechanism: chargepolarization-induced spin polarization determined in spin Hall devices evince that Hall voltages for $\mathbf{P r o}_{8} \mathbf{P Z n}_{1-3}$ substantially exceed that determined for the Pro $_{8}$ control and increase dramatically as the conjugation length of the achiral $\mathbf{P Z} \mathbf{n}_{n}$ component increases. Similarly, mC-AFM data show that the spin selectivity increases with the increasing $\operatorname{Pro}_{8} \mathbf{P Z n} \mathbf{n}_{1-3}$ length scale, highlighting that the difference between the effective barriers for the conduction of the two spin states of the electrons is amplified with augmented $\mathbf{P Z \mathbf { n } _ { n }}$ polarizability. Because of these effects, spin-dependent electrochemical data demonstrate that spin-polarized currents, which trace their genesis to the chiral Pro $_{8}$ moiety, propagate with no apparent dephasing over the augmented $\mathbf{P r o}_{8} \mathbf{P Z n}_{n}$ length scales, demonstrating that spin currents may be transmitted over molecular distances (e.g., $\mathbf{P Z n}_{3} \approx 35 \AA$ ) that greatly exceed the length of the chiral moiety $\left(\mathbf{P r o}_{8} \approx 15 \AA\right.$ ) responsible for inducing the CISS effect. Furthermore, because spin-polarized currents, realized by the CISS mechanism, have until now been generated and propagated only through chiral molecular insulators, this work underscores the exceptional properties of $\mathbf{P Z \mathbf { n } _ { n }}$ oligomers: because these compositions manifest (i) apparent coherent charge transport where tunneling, resonance, and hopping processes all contribute to current mediation ${ }^{4,5,23,24}$ and (ii) long spin relaxation times, ${ }^{22}$ these and related structures define compelling platforms for molecular spintronics applications.

\section{ASSOCIATED CONTENT}

\section{Supporting Information}

The Supporting Information is available free of charge on the ACS Publications website at DOI: 10.1021/jacs.9b06142.

Additional details regarding materials and methods, including the synthesis and characterization of molecules, the fabrication of Hall devices, and the preparation and characterization of self-assembled monolayers (PDF)

\section{AUTHOR INFORMATION}

\section{Corresponding Authors}

*ron.naaman@weizmann.ac.il

*michael.therien@duke.edu

ORCID

George Bullard: 0000-0002-3725-9046 
Chih-Hung Ko: 0000-0002-0261-8547

Ruobing Wang: 0000-0002-2414-8777

Suryakant Mishra: 0000-0002-9331-760X

Ron Naaman: 0000-0003-1910-366X

Michael J. Therien: 0000-0003-4876-0036

\section{Notes}

The authors declare no competing financial interest.

\section{ACKNOWLEDGMENTS}

F.T., S.M., A.K.M., and R.N. acknowledge the BSF-NSF through grant number 2015689 and the VW and Minerva Foundations for research support. G.B., C.-H.K., R.W., and M.J.T. acknowledge the National Science Foundation (DMR1610758) for funding this research. G.B. gratefully acknowledges the Fitzpatrick Institute of Photonics at Duke University for a John T. Chambers Scholars Award. The authors thank Prof. Dewey McCafferty for his advice regarding polypeptide synthesis.

\section{REFERENCES}

(1) Barraud, C.; Seneor, P.; Mattana, R.; Fusil, S.; Bouzehouane, K.; Deranlot, C.; Graziosi, P.; Hueso, L. E.; Bergenti, I.; Dediu, V.; Petroff, F.; Fert, A. Unravelling the Role of the Interface for Spin Injection into Organic Semiconductors. Nat. Phys. 2010, 6, 615-620.

(2) Daughton, J. M. Magnetic Tunneling Applied to Memory. J. Appl. Phys. 1997, 81, 3758-3763.

(3) Ciudad, D.; Gobbi, M.; Kinane, C. J.; Eich, M.; Moodera, J. S.; Hueso, L. E. Sign Control of Magnetoresistance Through Chemically Engineered Interfaces. Adv. Mater. 2014, 26, 7561-7567.

(4) Susumu, K.; Frail, P. R.; Angiolillo, P. J.; Therien, M. J. Conjugated Chromophore Arrays with Unusually Large Hole Polaron Delocalization Lengths. J. Am. Chem. Soc. 2006, 128, 8380-8381.

(5) Rawson, J.; Angiolillo, P. J.; Therien, M. J. Extreme Electron Polaron Spatial Delocalization in $\pi$-Conjugated Materials. Proc. Natl. Acad. Sci. U. S. A. 2015, 112, 13779-13783.

(6) Lin, V. S.-Y.; DiMagno, S. G.; Therien, M. J. Highly Conjugated, Acetylenyl Bridged Porphyrins: New models for Light-Harvesting Antenna Systems. Science 1994, 264, 1105-1111.

(7) Shediac, R.; Gray, M. H. B.; Uyeda, H. T.; Johnson, R. C.; Hupp, J. T.; Angiolillo, P. J.; Therien, M. J. Singlet and Triplet Excited States of Emissive, Conjugated Bis(porphyrin) Compounds Probed by Optical and EPR Spectroscopic Methods. J. Am. Chem. Soc. 2000, 122, 7017-7033.

(8) Duncan, T. V.; Susumu, K.; Sinks, L. E.; Therien, M. J. Exceptional Near-Infrared Fluorescence Quantum Yields and ExcitedState Absorptivity of Highly Conjugated Porphyrin Arrays. J. Am. Chem. Soc. 2006, 128, 9000-9001.

(9) Frail, P. R.; Susumu, K.; Huynh, M.; Fong, J.; Kikkawa, J. M.; Therien, M. J. Modulation of Dark Conductivity Over a $1 \times 10^{-12}$ to $1 \times 10^{-5} \mathrm{~S} / \mathrm{cm}$ Range Through Ancillary Group Modification in Amorphous Solids of Ethyne-Bridged (Porphinato)Zinc(II) Oligomers. Chem. Mater. 2007, 19, 6062-6064.

(10) Fisher, J. A. N.; Susumu, K.; Therien, M. J.; Yodh, A. G. Oneand Two-Photon Absorption of Highly Conjugated Multiporphyrin Systems in the Two-Photon Soret Transition Region. J. Chem. Phys. 2009, 130, 134506.

(11) Sanvito, S. Molecular Spintronics: The Rise of Spinterface Science. Nat. Phys. 2010, 6, 562-564.

(12) Ray, K.; Ananthavel, S. P.; Waldeck, D. H.; Naaman, R. Asymmetric Scattering of Polarized Electrons by Organized Organic Films of Chiral Molecules. Science 1999, 283, 814-816.

(13) Göhler, B.; Hamelbeck, V.; Markus, T. Z.; Kettner, M.; Hanne, G. F.; Vager, Z.; Naaman, R.; Zacharias, H. Spin Selectivity in Electron Transmission Through Self-Assembled Monolayers of Double-Stranded DNA. Science 2011, 331, 894-897.
(14) Xie, Z.; Markus, T. Z.; Cohen, S. R.; Vager, Z.; Gutierrez, R.; Naaman, R. Spin Specific Electron Conduction through DNA Oligomers. Nano Lett. 2011, 11, 4652-4655.

(15) Naaman, R.; Waldeck, D. H. Spintronics and Chirality: Spin Selectivity in Electron Transport through Chiral Molecules. Annu. Rev. Phys. Chem. 2015, 66, 263-281.

(16) Mathew, S. P.; Mondal, P. C.; Moshe, H.; Mastai, Y.; Naaman, R. Non-Magnetic Organic/Inorganic Spin Injector at Room Temperature. Appl. Phys. Lett. 2014, 105, 242408.

(17) Abendroth, J. M.; Nakatsuka, N.; Ye, M.; Kim, D.; Fullerton, E. E.; Andrews, A. M.; Weiss, P. S. Analyzing Spin Selectivity in DNAMediated Charge Transfer via Fluorescence Microscopy. ACS Nano 2017, 11, 7516-7526.

(18) Zwang, T. J.; Hürlimann, S.; Hill, M. G.; Barton, J. K. HelixDependent Spin Filtering through the DNA Duplex. J. Am. Chem. Soc. 2016, 138, 15551-15554.

(19) Aragonès, A. C.; Medina, E.; Ferrer-Huerta, M.; Gimeno, N.; Teixidó, M.; Palma, J. L.; Tao, N.; Ugalde, J. M.; Giralt, E.; DíezPérez, I.; Mujica, V. Measuring the Spin-Polarization Power of a Single Chiral Molecule. Small 2017, 13, 1602519.

(20) Mishra, D.; Markus, T. Z.; Naaman, R.; Kettner, M.; Göhler, B.; Zacharias, H.; Friedman, N.; Sheves, M.; Fontanesi, C. SpinDependent Electron Transmission through Bacteriorhodopsin Embedded in Purple Membrane. Proc. Natl. Acad. Sci. U. S. A. 2013, 110, 14872-14876.

(21) Maslyuk, V. V.; Gutierrez, R.; Dianat, A.; Mujica, V.; Cuniberti, G. Enhanced Magnetoresistance in Chiral Molecular Junctions. J. Phys. Chem. Lett. 2018, 9, 5453-5459.

(22) Rawson, J.; Angiolillo, P. J.; Frail, P. R.; Goodenough, I.; Therien, M. J. Electron Spin Relaxation of Hole and Electron Polarons in $\pi$-Conjugated Porphyrin Arrays: Spintronic Implications. J. Phys. Chem. B 2015, 119, 7681-7689.

(23) Li, Z.; Park, T.-H.; Rawson, J.; Therien, M. J.; Borguet, E. Quasi-Ohmic Single Molecule Charge Transport through Highly Conjugated meso-to-meso Ethyne-Bridged Porphyrin Wires. Nano Lett. 2012, 12, 2722-2727.

(24) Bruce, R. C.; Wang, R.; Rawson, J.; Therien, M. J.; You, W. Valence Band Dependent Charge Transport in Bulk Molecular Electronic Devices Incorporating Highly Conjugated Multi[(Porphinato)Metal] Oligomers. J. Am. Chem. Soc. 2016, 138, 2078-2081.

(25) Kumar, A.; Capua, E.; Vankayala, K.; Fontanesi, C.; Naaman, R. Magnetless Device for Conducting Three-Dimensional Spin-Specific Electrochemistry. Angew. Chem., Int. Ed. 2017, 56, 14587-14590.

(26) Kumar, A.; Capua, E.; Kesharwani, M. K.; Martin, J. M. L.; Sitbon, E.; Waldeck, D. H.; Naaman, R. Chirality-Induced Spin Polarization Places Symmetry Constraints on Biomolecular Interactions. Proc. Natl. Acad. Sci. U. S. A. 2017, 114, 2474-2478.

(27) Traub, W.; Shmueli, U. Structure of Poly-L-Proline I. Nature 1963, 198, 1165-1166.

(28) Mishra, S.; Poonia, V. S.; Fontanesi, C.; Naaman, R.; Fleming, A. M.; Burrows, C. J. Effect of Oxidative Damage on Charge and Spin Transport in DNA. J. Am. Chem. Soc. 2019, 141, 123-126.

(29) Lewis, F. D.; Liu, J.; Weigel, W.; Rettig, W.; Kurnikov, I. V.; Beratan, D. N. Donor-Bridge-Acceptor Energetics Determine the Distance Dependence of Electron Tunneling in DNA. Proc. Natl. Acad. Sci. U. S. A. 2002, 99, 12536-12541.

(30) Malak, R. A.; Gao, Z.; Wishart, J. F.; Isied, S. S. Long-Range Electron Transfer Across Peptide Bridges: The Transition from Electron Superexchange to Hopping. J. Am. Chem. Soc. 2004, 126, $13888-13889$. 